\title{
Evaluation of the corrosion behavior of modern spent nuclear fuels under repository conditions
}

\author{
Philip Kegler, Martina Klinkenberg, Felix Brandt, Guido Deissmann, and Dirk Bosbach \\ Forschungszentrum Jülich GmbH, Institute of Energy and Climate Research - Nuclear Waste Management and \\ Reactor Safety (IEK-6), 52428 Jülich, Germany
}

Correspondence: Philip Kegler (p.kegler@fz-juelich.de)

Published: 10 November 2021

\begin{abstract}
In Germany it is planned to directly dispose spent nuclear fuel (SNF) from nuclear power plants together with other high-level radioactive wastes (HLW) from former SNF reprocessing (e.g., vitrified waste), in a deep geological repository for heat-generating wastes - the siting process for this repository was started in 2017 and is ongoing. Based on several decades of research, development, and demonstration (RD\&D) it is generally accepted at the technical and scientific level that direct disposal of HLW and SNF in deep mined geological repositories is the safest and most sustainable option (CEC, 2011; IAEA, 2004).

The current efforts to improve the performance and accident tolerance of fuels in nuclear power generation resulted in an increased utilization of a variety of new types of light-water reactor (LWR) fuels such as fuels doped with $\mathrm{Cr}, \mathrm{Al}$, and Si. This doping leads to a significant change of the microstructure of the fuel matrix. The corrosion behavior of these types of fuels under conditions relevant to deep geological disposal has hardly been studied so far; however, this is of crucial importance as the development of a robust safety case for deep geological disposal of SNF requires a solid understanding of its dissolution behavior over very long time scales (up to 1 million years). To fill this knowledge gap, additional systematic studies on modern doped $\mathrm{UO}_{2}$ fuels
\end{abstract} were needed.

Corrosion experiments with SNF cannot entirely unravel all of the various concurring effects of the dissolution mechanism due to the chemical and structural complexity of SNF and its high beta and gamma radiation field during the first 1000 years; moreover, technical restrictions only allow a very limited number of experiments. Therefore, within the EU-DisCo project (https://www.disco-h2020.eu, last access: 11 October 2021), a very ambitious programme of corrosion studies on irradiated $\mathrm{Cr}$ and $\mathrm{Al} / \mathrm{Cr}$ doped fuels was carried out, which was complemented by systematic single-effect dissolution studies (e.g., with respect to doping level, grain size and thermodynamic aspects) performed on carefully prepared and characterized, simplified $\mathrm{UO}_{2}$-based model materials.

Here, we present recent results on the dissolution behavior of tailor-made $\mathrm{UO}_{2}$ model materials in accelerated static batch experiments using $\mathrm{H}_{2} \mathrm{O}_{2}$ as simulant for radiolytic oxidants, present in long-term disposal scenarios for SNF in failed container conditions due to the alpha irradiation of water. In these dissolution experiments pure $\mathrm{UO}_{2}$ reference pellets exhibiting different densities and grain sizes, as well as $\mathrm{Cr}$-doped $\mathrm{UO}_{2}$ pellets with various Cr-doping levels, produced using different doping methods having different grain sizes, were used. In addition, $\mathrm{Nd}$-doped and industrially produced $\mathrm{Cr}$ - and $\mathrm{Cr} / \mathrm{Nd}$-doped $\mathrm{UO}_{2}$ pellets were used to determine the influence of these parameters on the dissolution rates. The dissolution experiments were performed under strictly controlled conditions with respect to exclusion of oxygen, temperature control, and exclusion of light.

This bottom-up approach was followed to understand how the addition of Cr-oxide into the fuel matrix affects SNF dissolution behavior under repository relevant conditions. The results of the dissolution experiments performed with real SNF and the model materials obtained by the DisCo partners build the basis for numerical simulations on the dissolution behavior of modern SNF. First results of the data evaluation indicate that the addition of dopants and the consequential modification of the fuel matrix does not lead to a significant change 
of the dissolution behavior of these fuels under repository relevant conditions compared to standard SNF (i.e. dissolution rates agree within an order of magnitude).

Kurzfassung. In Deutschland ist geplant, abgebrannte Kernbrennstoffe (SNF [,,spent nuclear fuel“]) aus Kernkraftwerken zusammen mit anderen hochradioaktiven Abfällen (HLW [,high-level radioactive wastes"]) aus der ehemaligen SNF-Wiederaufbereitung (z. B. verglaste Abfälle) direkt in einem geologischen Tiefenlager für hitzeentwickelnde Abfälle zu deponieren - die Standortsuche für dieses Endlager wurde 2017 begonnen und ist noch nicht abgeschlossen. Basierend auf mehreren Jahrzehnten Forschung, Entwicklung und Demonstration (RD\&D [,research, development, and demonstration“]) ist auf technischer und wissenschaftlicher Ebene allgemein anerkannt, dass die direkte Endlagerung von HLW und SNF in geologischen Tiefenlagern die sicherste und nachhaltigste Option darstellt (CEC, 2011; IAEA, 2004).

Die aktuellen Bemühungen, die Leistung und Störfalltoleranz von in der Kernenergiegewinnung genutzten Brennstoffen zu verbessern, führten zur verstärkten Verwendung einer Vielzahl neuer Brennstoffarten für Leichtwasserreaktoren (LWR), wie beispielsweise von mit $\mathrm{Cr}$, Al und Si dotierten Brennstoffe. Durch diese Additive kommt es zu signifikanten Veränderungen der Mikrostruktur der Brennstoffmatrix. Das Korrosionsverhalten derartiger Brennstoffe unter geologischen Tiefenlagerbedingungen ist bisher kaum untersucht. Dies ist jedoch von entscheidender Bedeutung, da die Entwicklung eines robusten Sicherheitsnachweises für geologische Tiefenlager von SNF ein umfassendes Verständnis der Auflösung dieser Abfälle über sehr lange Zeiträume (bis zu 1 Mio. Jahre) erfordert. Um diese Wissenslücke zu schließen, waren zusätzliche systematische Studien zu modernen dotierten $\mathrm{UO}_{2}$-Brennstoffen erforderlich.

Aufgrund der chemischen und strukturellen Komplexität sowie des hohen Beta- und Gammastrahlungsfeldes von SNF während der ersten 1000 Jahre, können Korrosionsexperimente mit abgebrannten Kernbrennstoffe nicht alle konkurrierenden Effekte der Auflösungsmechanismen entwirren. Daher wurde im Rahmen des EU-DisCoProjekts (https://www.disco-h2020.eu, letzter Zugriff: 11. Oktober 2021) ein sehr ambitioniertes Programm von Korrosionsstudien an bestrahlten $\mathrm{Cr}$ - und $\mathrm{Al} / \mathrm{Cr}$ dotierten Brennstoffen durchgeführt, das durch systematische Einzeleffektzerfallsstudien (z. B. bezüglich Additivmenge, Korngröße und thermodynamischen Aspekten) an sorgfältig präparierten und charakterisierten, vereinfachten $\mathrm{UO}_{2}$-basierten Modellmaterialien ergänzt wurde.

Hier präsentieren wir aktuelle Ergebnisse zum Auflösungsverhalten maßgeschneiderter $\mathrm{UO}_{2}$-Modellmaterialien in beschleunigten statischen BatchExperimenten unter Verwendung von $\mathrm{H}_{2} \mathrm{O}_{2}$ als Vertreter für radiolytische Oxidationsmittel, die in langfristigen Entsorgungsszenarien für SNF bei undichten Behältern aufgrund der Alpha-Bestrahlung von Wasser zu berücksichtigen sind. Bei diesen Auflösungsversuchen kamen reine $\mathrm{UO}_{2}$-Referenzpellets mit unterschiedlichen Dichten und Korngrößen sowie $\mathrm{UO}_{2}$ Pellets mit Cr-Dotierung mit divergenten Dotierungsmengen, hergestellt mittels verschiedener Verfahren und mit variierenden Korngrößen, zum Einsatz. Darüber hinaus wurden $\mathrm{UO}_{2}$-Pellets mit Nd-Additiv und industriell hergestellte $\mathrm{UO}_{2}$-Pellets mit $\mathrm{Cr}-$ und $\mathrm{Cr} / \mathrm{Nd}$-Dotierung verwendet, um den Einfluss dieser Parameter auf die Auflösungsgeschwingigkeit zu ermitteln. Die Auflösungsexperimente wurden unter streng kontrollierten Bedingungen bezüglich Sauerstoffausschluss, Temperaturkontrolle und Lichtausschluss durchgeführt.

Dieser von „unten nach oben“ ausgerichtete Ansatz (Bottom-up-Ansatz) wurde verfolgt, um zu verstehen, wie die Zugabe von Cr-Oxid in die Brennstoffmatrix das SNF-Auflösungsverhalten unter endlagerrelevanten Bedingungen beeinflusst. Die Ergebnisse der mit realen SNF durchgeführten diesbezüglichen Experimente und die von den DisCo-Partnern erhaltenen Modellmaterialien bilden die Grundlage für numerische Simulationen zum Ablauf des Zerfalls moderner SNF.

Laut erster Ergebnisse der Datenauswertung haben die Zugabe von Additiven und die damit verbundene Modifikation der Brennstoffmatrix keine signifikante Veränderung der Auflösung dieser Brennstoffe unter endlagerrelevanten Bedingungen im Vergleich zu Standard-SNF zur Folge (d. h. Auflösungsraten stimmen innerhalb einer Größenordnung überein). 
Financial support. This project has received funding from the European Union's Horizon 2020 research and training programme of the European Atomic Energy Community (EURATOM) (H2020NFRP2016/2017) under grant agreement no. 755443 (DisCo).

\section{References}

CEC - Council of the European Union: Council directive 011/70/EURATOM of 19 July 2011 Establishing a Community Framework for the Responsible and Safe Management of Spent Fuel and Radioactive Waste, CEC, Brussels, Belgium, 2011.
IAEA - International Atomic Energy Agency: Scientific and Technical Basis for the Geological Disposal of Radioactive Wastes, Technical Reports Series 413, IAEA, Vienna, Austria, 2004. 